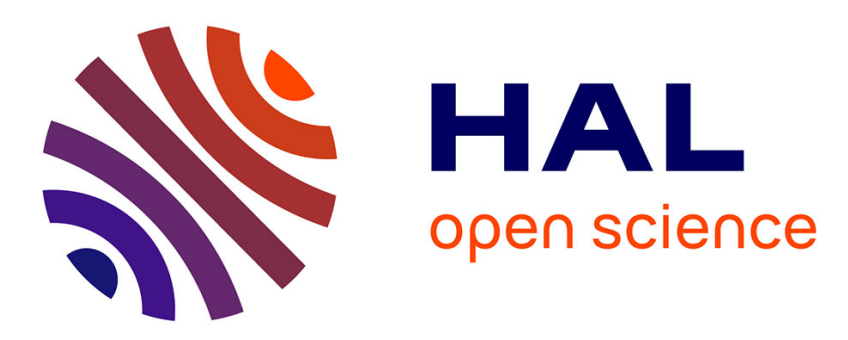

\title{
La concurrence imparfaite entre les intermédiaires financiers est-elle toujours néfaste à la croissance économique?
}

Jean-Bernard Chatelain, Bruno Amable

\section{- To cite this version:}

Jean-Bernard Chatelain, Bruno Amable. La concurrence imparfaite entre les intermédiaires financiers est-elle toujours néfaste à la croissance économique?. Revue Economique, 1996, 3, pp.765-775. halshs$00009024 \mathrm{v} 2$

\section{HAL Id: halshs-00009024 \\ https://shs.hal.science/halshs-00009024v2}

Submitted on 5 Dec 2006

HAL is a multi-disciplinary open access archive for the deposit and dissemination of scientific research documents, whether they are published or not. The documents may come from teaching and research institutions in France or abroad, or from public or private research centers.
L'archive ouverte pluridisciplinaire HAL, est destinée au dépôt et à la diffusion de documents scientifiques de niveau recherche, publiés ou non, émanant des établissements d'enseignement et de recherche français ou étrangers, des laboratoires publics ou privés. 


\title{
La concurrence imparfaite entre les intermédiaires financiers est-elle toujours néfaste à la croissance économique ?
}

\author{
Bruno Amable* \\ Jean-Bernard Chatelain**
}

\begin{abstract}
Les performances économiques de l'Allemagne et du Japon laissent supposer qu'une faible concurrence entre les intermédiaires financiers n'est pas nécessairement un obstacle à la croissance. Cet article présente un modèle de croissance endogène comportant un secteur d'intermédiation financière en concurrence imparfaite. Une forte concentration du secteur bancaire implique une marge d'intermédiation financière importante. Elle exerce un effet négatif sur la croissance par une baisse de la rémunération de l'épargne et une hausse du coût du capital. Mais les désavantages de la concurrence imparfaite peuvent être plus que compensés par l'accroissement de la productivité des investissements des entreprises provenant de l'expertise et du contrôle exercé par des banques " universelles ».
\end{abstract}

\section{GROWTH WITH IMPERFECTLY COMPETITIVE FINANCIAL INTERMEDIATION}

This paper presents a model of endogenous growth with an imperfectly competitive financial intermediation sector. A concentrated banking sector implies a higher level of intermediation margin, which exerts a depressive influence on savings and investment. This prediction alone would suggest that the high growth performance of Germany and Japan are not related to the particular features of their financial sectors. However, if large financial institutions exert a positive influence on the productivity of industrial investment, thanks to their expertise and ability to advise entrepreneurs, the disadvantages of imperfect competition in terms of growth may be more than offset by the increase in productivity. The paper shows that this can be the case in a simple model of endogenous growth.

Classification JEL : 016

* INRA et CEPREMAP, 65 boulevard de Brandebourg, 94205 Ivry Cedex France.

** Banque de France, Centre de Recherche, 39 rue Croix-des-Petits-Champs, 75049 Paris Cedex 01.

Les auteurs remercient, sans les impliquer, Thierry Magnac, Pierre Sicsic, Bernard Bensaid, Henri Pagès, Yishay Yafeh et Oved Yosha pour leurs commentaires et suggestions. Les opinions émises n'engagent pas la Banque de France. 


\section{INTRODUCTION}

L'influence des intermédiaires financiers sur la croissance économique a fait l'objet d'un nouvel examen dans des articles récents ${ }^{1}$. Le secteur financier joue un rôle important en diminuant les coûts de transactions, en facilitant la diversification des risques, en mobilisant l'épargne et en allouant de manière efficace ces ressources aux emprunteurs. Des travaux empiriques confirment que le développement financier exerce une influence importante sur la croissance économique ${ }^{2}$. Cependant, la plupart de ces contributions récentes n'ont pas cherché à comparer les avantages respectifs des différents types de systèmes financiers vis-à-vis de la croissance. Parmi les pays développés, une distinction maintenant classique oppose les systèmes financiers à prédominance bancaire (Japon et Allemagne) aux systèmes financiers fondés sur les marchés (modèle anglosaxon) (Mayer [1988]). Cette question a initialement été soulevée par Gerschenkron [1962] en ce qui concerne la croissance de l'Allemagne entre 1870 et 1914, alors que le Royaume-Uni commençait à connaître un déclin relatif. Il attribua aux banques « universelles » qui financèrent les grandes entreprises industrielles allemandes un rôle prépondérant pour expliquer ce phénomène. Cet argument est également discuté pour expliquer la croissance allemande ou japonaise dans l'après-guerre (Edwards et Fischer [1994]). Les banques « universelles » allemandes et les banques « principales » japonaises sont plus impliquées dans la gestion et le contrôle des entreprises que les banques octroyant des crédits sans prise de participations sauf en cas de faillite. Les banques « universelles » collectent des dépôts et sont simultanément prêteuses et actionnaires. Elles ont un ou plusieurs représentants au conseil d'administration des entreprises emprunteuses, ce qui leur donne un grand pouvoir de contrôle. " L'opinion la plus répandue ${ }^{3}$ » en la matière est que le rôle de conseil et de contrôle joué par les banques allemandes explique les performances macroéconomiques de ce pays.

Durant la période 1870-1914, les systèmes bancaires anglais et allemands connurent un mouvement de concentration important. Le nombre de banques au Royaume Uni passa de 386 en 1870 à 112 en 1910 (Capie et Webber [1985]). En Allemagne, sur l'ensemble de la période, six grandes banques fournissaient la majorité des services financiers aux grandes entreprises industrielles, et, en 1913, trois d'entre elles étaient les plus grandes entreprises du pays ${ }^{4}$. Dans ce même pays, la concurrence des marchés financiers est restée limitée, car l'émission de titres ou d'obligations était principalement contrôlée par les banques universelles. Au cours de la même période, les banques universelles ont modifié leurs sources de financement : la part de l'actionnariat diminue rapidement par rapport à celle de la collecte de dépôts (Da Rin [1993]). Les banques universelles étaient donc dans la situation de prélever des rentes de monopole sur leurs emprunteurs et, dans une moindre mesure, leurs déposants, tant que ceux-ci dis-

1. Voir Amable et Chatelain [1995] pour une revue de littérature.

2. Levine et Renelt [1991], King et Levine [1993], Berthélémy et Varoudakis [1994], Neusser et Kugler [1994].

3. L'expression est tirée de Edwards et Fisher [1994]. Il s'agit de l'opinion selon laquelle le système allemand de banque universelle est supérieur au système britannique.

4. Tilly [1992], p. 94. 
posaient de peu d'opportunités de se financer à l'étranger. À cette même période, le système bancaire britannique était lui-même concentré, mais ces banques ne remplissaient pas le rôle des banques universelles. On s'attendrait à ce que les effets de la concurrence imparfaite dans le secteur bancaire soient un frein au développement économique. Cependant, l'Allemagne connut un développement industriel considérable au cours de cette période, tandis que l'Angleterre subissait un déclin industriel relatif.

La concurrence imparfaite entre les intermédiaires financiers n'aurait pas été à cette occasion un obstacle au développement. Cette observation est en contradiction avec des modélisations récentes des effets sur la croissance de la concurrence imparfaite entre les intermédiaires (Berthélémy et Varoudakis [1994], Zilibotti [1994]). Ces deux articles montrent que la concurrence imparfaite peut diminuer le taux de croissance de long terme d'un équilibre « haut ", mais aussi maintenir l'économie dans un piège de sous-développement (équilibre «bas »), du fait des coûts fixes dans le secteur d'intermédiation et de l'emploi d'une proportion d'un facteur de production (le travail dans Berthélémy et Varoudakis [1994]) dans cette activité non directement productive.

Cet article propose un arbitrage entre les effets négatifs de la concurrence imparfaite et l'activité de contrôle des banques. Nous reprenons l'hypothèse que l'activité d'intermédiation a lieu dans des conditions de concurrence imparfaite entre les banques. Mais ce coût fixe, à la différence des articles de Berthélémy et Varoudakis [1994] et de Zilibotti [1994] ne représente pas uniquement des coûts de fonctionnement sans effets bénéfiques sur d'autres secteurs de l'économie. Une partie des frais de fonctionnement des banques correspond à des emplois bancaires consacrés à l'activité d'expertise et conseil, de sélection puis de contrôle des entreprises. Plus ce coût sera élevé, plus la productivité des entreprises augmentera, avec comme contrepartie une moindre concurrence entre les intermédiaires financiers. Il est possible que les gains de productivité provenant de la technologie de contrôle des intermédiaires excède la hausse des coûts provenant d'une moindre concurrence entre les intermédiaires (i.e. la hausse du coût du capital et la baisse de la rémunération de l'épargne). Dans ce cas, un système bancaire peu concurrentiel peut quand même se révéler suffisamment efficace pour la croissance de long terme. Cette situation favorable n'est pas possible lorsque le coût de contrôle est très élevé (la technologie de contrôle est peu efficace) : dans ce cas, les effets négatifs standards de la concurrence imparfaite prédominent.

L'article est organisé comme suit : la section 2 présente un modèle de croissance endogène avec concurrence monopolistique la Cournot entre les intermédiaires financiers. La section 3 compare les taux de croissance en fonction de la technologie de contrôle des entreprises par les intermédiaires financiers. La section 4 conclut l'article et suggère des voies d'approfondissement.

\section{LE MODÈLE}

\section{Production}

Nous présentons un modèle simple de croissance avec un secteur d'intermédiation financière caractérisée par une concurrence imparfaite. L'économie est constituée d'une population de masse 1 sur un continuum de taille fixe avec des 
générations imbriquées d'agents vivant deux périodes. Les travailleurs sont dotés d'une unité de travail qu'ils offrent de façon inélastique au cours de la première période de leur vie. Ils peuvent travailler dans le secteur du bien final ou dans le secteur d'intermédiation financière. Ils se soucient de la consommation au cours des deux périodes de leur vie et ont une fonction d'utilité $u_{w}\left(c_{1}, c_{2}\right)$. À des fins de simplification, on suppose que leur maximisation d'utilité sous contrainte budgétaire conduit à une fonction d'épargne qui est linéaire avec le revenu de première période et isoélastique par rapport au taux d'intérêt servi sur les dépôts ${ }^{1}$. En notant $\mathrm{W}_{t}$ la masse salariale agrégée, l'épargne agrégée $\mathrm{S}_{t}$ est alors :

$$
\mathrm{S}_{t}=s \cdot \mathrm{R}_{d}^{\sigma} \cdot \mathrm{W}_{t}
$$

avec $\mathrm{R}_{d}$ le taux d'intérêt sur les dépôts ${ }^{2}$.

La fonction de production du secteur du bien final est :

$$
\mathrm{Y}_{t}=\mathrm{A}_{t} \cdot \mathrm{K}_{t}^{\theta} \cdot \mathrm{L}_{t}^{1-\theta}
$$

$\mathrm{K}_{t}$ est le stock de capital agrégé et $\mathrm{L}_{t}$ est le montant de travail affecté au secteur final (1 - $\mathrm{L}_{t}$ est donc le travail affecté au secteur d'intermédiation). De plus, on suppose que

$$
\mathrm{A}_{t}=\mathrm{K}_{t}^{1-\theta}
$$

Ainsi la production exhibe-t-elle des rendements constants par rapport au capital. Le terme d'externalité $\mathrm{A}_{t}$ permet à la fois une croissance endogène et la concurrence parfaite sur le marché des biens (Romer [1986]).

Les agents naissent sans autre dotation que leur travail. Les firmes doivent emprunter des financements externes auprès des banques pour mettre en œuvre leurs projets d'investissement. Les firmes sont dotées d'un projet qui leur permet de transformer une unité de produit final en $z$ unités de capital à la période suivante. En notant $\mathrm{E}_{t}$ le montant agrégé emprunté par les firmes, on aura donc $\mathrm{K}_{t+1}=z$. $\mathrm{E}_{t}$. Une firme emploie alors $l_{t+1}$, produit du bien final, et rem-

1. On peut associer une fonction de dépense à un tel comportement d'épargne. Si $p_{1}$ et $p_{2}$ sont les prix de première et deuxième période respectivement, la dépense associée à la fonction d'épargne $\mathrm{S}=w \cdot\left(\frac{R}{p_{2}}\right)^{\sigma} \cdot c\left(p_{1}\right)$ est $e\left(p_{1}, p_{2}\right)=p_{1} \cdot \exp \left[\frac{-\mathrm{K}}{\sigma} \cdot\left(\frac{\mathrm{R} \cdot p_{1}}{p_{2}}\right)^{\sigma}\right]$.

La fonction de dépense est homogène de degré 1 en $\left(p_{1}, \frac{p_{2}}{\mathrm{R}}\right)$, croissante en $p_{2}$ pour $\sigma>0$, croissante en $p_{1} \quad$ quand $\quad 1-K \cdot\left(\frac{p_{2}}{\mathrm{R}}\right)^{-\sigma} \cdot p_{1}^{\sigma}>0$. De plus, $\left(\frac{\partial^{2} e}{\partial p_{1}^{2}}\right) \cdot\left(\frac{\partial^{2} e}{\partial p_{2}^{2}}\right)-\left(\frac{\partial^{2} e}{\partial_{p 1} \cdot \partial_{p 2}}\right)^{2}=0$.

2. Seuls les sentiers de croissance à taux d'intérêt constant sont retenus. 
bourse l'emprunt. En notant $w_{t+1}$ le taux de salaire et $\mathrm{R}$ le facteur d'intérêt sur les prêts, la maximisation de profit des entreprises conduit à :

$$
\left(\mathrm{E}_{t}, \mathrm{~L}_{t}\right) \in \operatorname{ArgMax}\left\{\mathrm{A}_{t+1} \cdot z^{\theta} \cdot \mathrm{E}_{t}^{\theta} \cdot \mathrm{L}_{t+1}^{1-\theta}-\mathrm{R} \cdot \mathrm{E}_{t}-w_{t+1} \cdot \mathrm{L}_{t+1}\right\}
$$

Par conséquent, le taux de salaire et la demande de prêts sont :

$$
w_{t+1}=\mathrm{A}_{t+1} \cdot z^{\theta} \cdot(1-\theta) \cdot \mathrm{E}_{t}^{\theta} \cdot \mathrm{L}_{t+1}^{-\theta}
$$

et

$$
\mathrm{E}_{t}=\mathrm{R}^{\frac{1}{\theta-1}} \cdot \mathrm{L}_{t+1} \cdot z^{\frac{\theta}{1-\theta}} \cdot\left(\mathrm{A}_{t+1} \cdot \theta\right)^{\frac{1}{1-\theta}}
$$

La demande de fonds décroît avec le facteur d'intérêt $\mathrm{R}$, est proportionnelle au travail employé dans la production de bien final à la période suivante et crôit avec la productivité des projets d'investissement $z$.

\section{Le secteur d'intermédiation financière}

Le secteur financier est composé de $\mathrm{N}$ banques symétriques en concurrence à la Cournot. Les banques collectent l'épargne des jeunes travailleurs et accordent des prêts aux firmes. La concurrence est imparfaite sur les marchés des dépôts et des prêts. Chaque banque maximise ses profits période par période en considérant fixé le comportement des autres banques. Le fonctionnement d'une banque exige que $b$ travailleurs soient engagés, quel que soit le niveau d'activité de la banque. Chaque banque supporte donc un coût fixe de travail et un coût variable sous la forme du taux d'intérêt payé sur les dépôts. Les banques maximisent leurs profits sous contrainte d'équilibre entre dépôts et crédits :

$$
\mathrm{R} \cdot \frac{\mathrm{E}_{t}}{\mathrm{~N}}-\mathrm{R}_{d} \cdot \frac{\mathrm{S}_{t}}{\mathrm{~N}}-w_{t+1} \cdot b,
$$

Elles collectent une fraction égale de l'épargne totale et accordent un montant égal de prêts. La maximisation de profits conduit à une marge d'intermédiation entre le coût des ressources pour la banque, $\mathrm{R}_{d}$, et le facteur d'intérêt sur les prêts $\mathbf{R}$.

$$
\mathrm{R} \cdot \frac{1+\frac{\theta-1}{\mathrm{~N}}}{1+\frac{1}{\mathrm{~N} \cdot \sigma}}=\mathrm{R}_{d}
$$

Par la suite, on notera $m(\mathrm{~N})=\frac{1+\frac{\theta-1}{\mathrm{~N}}}{1+\frac{1}{\mathrm{~N} \cdot \sigma}}$ l'inverse de la marge d'intermé-

diation. Elle augmente avec $\mathrm{N}$, le nombre de banques, de telle façon que la marge d'intermédiation est décroissante lorsque l'intensité de la concurrence dans le secteur financier augmente. La marge est décroissante en $\sigma$ l'élasticité de l'épargne au taux d'intérêt et en $\theta$ l'élasticité de la production au capital.

La marge d'intermédiation constitue un écart entre le coûts des fonds et le rendement de l'épargne. Cela tend à rendre les fonds prêtables plus chers et à diminuer la rémunération de l'épargne en comparaison de la situation qui pré- 


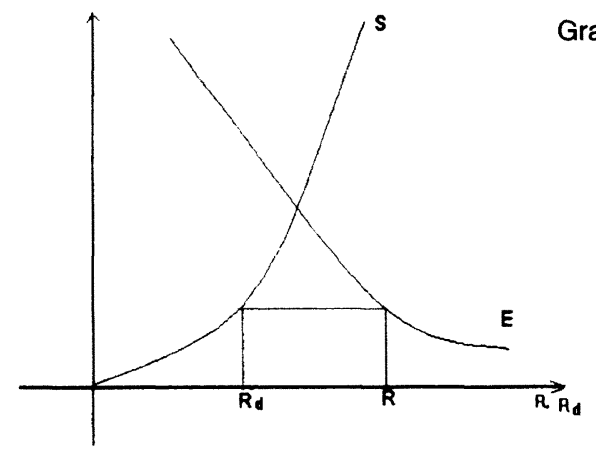

Graphique 1. L'équilibre épargne-emprunt

vaudrait en concurrence parfaite. Dans ce cas, l'égalité entre épargne et investissement se produirait à l'intersection des courbes $S$ et $E$ du graphique 1. Avec la concurrence imparfaite, l'équilibre épargne-emprunt se produit à un niveau inférieur. Ce seul effet va réduire le montant investi à chaque période avec pour conséquence un taux de croissance moins élevé, puisque la croissance est fondée sur l'accumulation de capital dans ce modèle.

Cependant, l'influence du secteur d'intermédiation financière sur la croissance ne se limite pas à la déterminantion des taux d'intérêt. Si une augmentation du nombre de banques diminue les distortions dues à la concurrence imparfaite, amenant par là même une hausse des niveaux de l'épargne et de l'emprunt, elle a aussi pour conséquence d'augmenter les coûts fixes d'intermédiation totaux. Les montants supplémentaires de ressources allouées à l'intermédiation financière ont un effet contraire sur la croissance.

Considérons maintenant une détermination endogène de la structure du secteur bancaire avec entrée libre. Le nombre de banques est, à l'équilibre, déterminé par une condition de profit nul :

$$
\mathrm{R} \cdot \frac{\mathrm{E}_{t}}{\mathrm{~N}}-\mathrm{R}_{d} \cdot \frac{\mathrm{S}}{\mathrm{N}}-w_{t+1} \cdot b=0
$$

Cette condition peut s'écrire :

$$
\theta \cdot[1+(1-\theta) \cdot \sigma] \cdot(1-b \cdot \mathbf{N})=b \cdot(1-\theta) \cdot \mathrm{N} \cdot(1+\sigma \cdot \mathrm{N})
$$

et le nombre de banques à l'équilibre est donné, en ignorant la contrainte de nombre entier, par :

$$
\mathrm{N}=\frac{-b \cdot[1+\sigma \cdot \theta \cdot(1-\theta)]+\sqrt{\begin{array}{c}
b^{2} \cdot[1+\sigma \cdot \theta \cdot(1-\theta)]^{2}+ \\
4 \cdot \sigma \cdot b \cdot \theta \cdot(1-\theta) \cdot[1+\sigma \cdot(1-\theta)]
\end{array}}}{2 \cdot b \cdot(1-\theta) \cdot \sigma}
$$

On peut vérifier que $\frac{\partial N}{\partial b}$ est du signe de $-\theta \cdot[1+\sigma \cdot(1-\theta)]$, et donc $\frac{\partial \mathrm{N}}{\partial b}<0$. Le nombre de banques, c'est-à-dire l'intensité de la concurrence dans le secteur d'intermédiation, décroît avec le coût fixe. Sans surprise, un secteur bancaire très concentré est associé à de hauts coûts d'établissement et à une marge d'intermédiation élevée. 
La taille relative du secteur financier peut s'apprécier avec l'emploi relatif de ce secteur, $\mathrm{N} \cdot b$. On peut vérifier que l'emploi dans le secteur d'intermédiation augmente avec le coût d'établissement $b$, malgré le lien négatif entre $b$ et le nombre de banques $\mathrm{N}$.

$$
\mathrm{N} \cdot b=\frac{\theta \cdot(1-m(\mathrm{~N}))}{1-\theta+\theta \cdot(1-m(\mathrm{~N}))}
$$

$\frac{\partial(\mathrm{N} \cdot b)}{\partial \mathrm{N}}$ est du signe de $-m^{\prime}(\mathrm{N})$, donc négatif. Comme $\frac{\partial \mathrm{N}}{\partial b}<0, \frac{\partial(\mathrm{N} \cdot b)}{\partial b}>0$.

On peut noter que le cas de la concurrence parfaite est le cas limite où $b=0$, ce qui donne un nombre de banque indéterminé. La marge d'intermédiation est égale à 0 .

\section{L'ÉQUILIBRE DYNAMIQUE}

L'équilibre se manifeste par l'égalité entre épargne et investissement et l'absence de différenciel de salaire entre les secteurs de la banque et du bien final. L'égalité entre investissement et épargne s'exprime comme :

$$
s \cdot z \cdot(\mathrm{R} \cdot m(\mathrm{~N}))^{\sigma} \cdot \mathrm{W}_{t}=\mathrm{K}_{t+1}
$$

En prenant en compte l'externalité dans la fonction de production dans l'expression de la demande de crédit, le taux d'intérêt sur le crédit s'écrit :

$$
\mathrm{R}=(1-\mathrm{N} \cdot b)^{1-\theta} \cdot z \cdot \theta
$$

De même, le salaire réel s'exprime comme : $\mathrm{W}_{t}=z \cdot(1-\theta) \cdot \mathrm{E}_{t} \cdot(1-\mathrm{N} \cdot b)^{-\theta}$, le facteur de croissance d'équilibre est donc :

$$
\mathrm{G}=\frac{\mathrm{K}_{t+1}}{\mathrm{~K}_{t}}=s \cdot(1-\theta) \cdot(1-\mathrm{N} \cdot b)^{\sigma(1-\theta)-\theta} \cdot m(\mathrm{~N})^{\sigma} \cdot z^{1+\sigma} \cdot \theta^{\sigma}
$$

Le taux de croissance de l'économie dépend de la structure du secteur bancaire à travers deux effets. La marge d'intermédiation dépend négativement du nombre de banques. Plus celui-ci est élevé, moins grandes seront les distorsions entre $\mathrm{R}$ et $\mathrm{R}_{d}$. Cet effet contribue à augmenter l'épargne et l'investissement et influence positivement la croissance par le terme en $m(\mathrm{~N})^{\sigma}$ dans l'équation précédente. Le deuxième effet est dû à l'allocation du travail entre les deux secteurs. La présence d'un nombre accru de banques implique une allocation du travail en faveur du secteur d'intermédiation, ce qui contribue à augmenter les salaires dans le secteur du bien final et affecte le niveau de l'épargne. Cet effet est négatif pour la croissance lorsque $\sigma>\frac{\theta}{1-\theta}$.

Le taux de croissance peut être réexprimé comme :

$$
\mathrm{G}=s \cdot(1-\theta) \cdot(1-\mathrm{N} \cdot b)^{-\theta} \cdot(1+\sigma) \cdot(\theta-\mathrm{N} \cdot b)^{\sigma} \cdot z^{1+\sigma}
$$

La taille du secteur financier $(\mathrm{N} \cdot b)$ a deux effets. Un positif à travers le terme en $(1-\mathrm{N} \cdot b)^{-\theta \cdot(1+\sigma)}$, l'autre négatif à travers le terme en $(\theta-\mathrm{N} \cdot b)^{\sigma} \cdot \frac{\partial \mathrm{G}}{\partial(\mathrm{N} \cdot b)}$ 
possède le signe de $\theta \cdot(1-\sigma) \cdot(\theta-N \cdot b)-\sigma \cdot(1-N \cdot b)$, qui est positif quand $\mathrm{N} \cdot b<\frac{\theta^{2}-\sigma \cdot\left(1-\theta^{2}\right)}{\theta-\sigma \cdot(1-\theta)}$. Au-delà d'un certain seuil, la taille du secteur financier exerce un effet négatif sur la croissance. Par conséquent, de hauts niveaux de coûts fixes amèneront une chute du taux de croissance de l'économie.

Un haut niveau du coût fixe $b$ est donc associé à un faible nombre de banques et une forte marge d'intermédiation, avec pour conséquence un faible taux de croissance. La seule prise en compte de ces effets indique qu'une concurrence accrue dans le secteur d'intermédiation, rendu possible par exemple par une diminution du coût d'accès à l'activité bancaire, a pour conséquence un taux de croissance plus élevé. Un tel résultat ne cadre pas avec l'opinion la plus répandue concernant l'apport positif qu'a constitué un secteur de grandes banques dans des pays comme l'Allemagne ou le Japon.

Cependant, les systèmes bancaires allemand ou japonais ne sont pas seulement caractérisés par la taille et le faible nombre de banques. Les grandes banques de ces pays sont supposées remplir des tâches que des banques de taille plus petite ne peuvent pas accomplir. Les banques à l'allemande sont tenues pour plus efficaces que les banques à l'anglo-saxonne ou un système de marchés financiers dans le domaine du contrôle de l'activité des firmes. Elles ont un contrôle significatif des droits de vote et peuvent placer leurs représentants dans les conseils de surveillance des firmes. La fonction de surveillance est d'autant plus aisée que les banques ont un droit de regard sur les décisions stratégiques des firmes. Un autre argument repose sur la capacité des banques à conseiller les firmes dans leurs projets industriels. L'expertise technique de la banque est d'autant plus grande que celle-ci couvre une large gamme d'activités industrielles. Donc, une grande taille permet de disposer d'une équipe de conseillers techniques qui évaluent les projets et conseillent les industriels, avec pour conséquence une augmentation de la productivité des projets d'investissement.

Pour illustrer cette possibilité, on spécifie une fonction croissante $z(b)$ avec $z$ atteignant une limite à l'infini. $z(b) \equiv \frac{6}{1+\exp [-10 \cdot b]}$, et on adopte les valeurs suivantes pour les coefficients : $s=0.5, \sigma=1.2$ et $\theta=0.4$. Le taux de croissance est alors :

$$
\mathrm{G}=s \cdot(1-\theta) \cdot(1-\mathrm{N} \cdot b)^{-\theta \cdot(1+\sigma)} \cdot(\theta-\mathrm{N} \cdot b)^{\sigma} \cdot z(b)^{1+\sigma}
$$

Le graphe de $\mathrm{G}(b)$ est donné en graphique 2 . Une hausse du coût fixe $b$ diminue le taux de croissance pour une concurrence presque parfaite ( $b$ petit) ou pour une concurrence très peu intense, proche du monopole ( $b$ grand), mais elle l'augmente pour des valeurs intermédiaires du fait de la hausse de $z$ qui compense les pertes dues aux coûts d'intermédiation. Donc, des économies possédant un secteur bancaire très concentré peuvent bénéficier de taux de croissance plus élevés malgré de forts coûts d'ntermédiation et de fortes distorsions.

Pour simplifier, supposons deux types de banques, deux coûts associés, $\underline{b}$ et $\bar{b}$, et deux types de projets correspondant à deux productivité $z$ et $\bar{z}, \underline{b}<\bar{b}$ et $z<\bar{z}$. Le coût $\bar{b}$ correspond à la présence d'une équipe de conseillers au sein de la banque, le coût $\underline{b}$ correspond à une simple activité d'intermédiation. Les deux types de projets ne subissent pas le même aléa. Quelle que soit la banque, le projet de type $z$ réussit toujours avec certitude, en revanche, le projet de taille $\bar{z}$ ne 


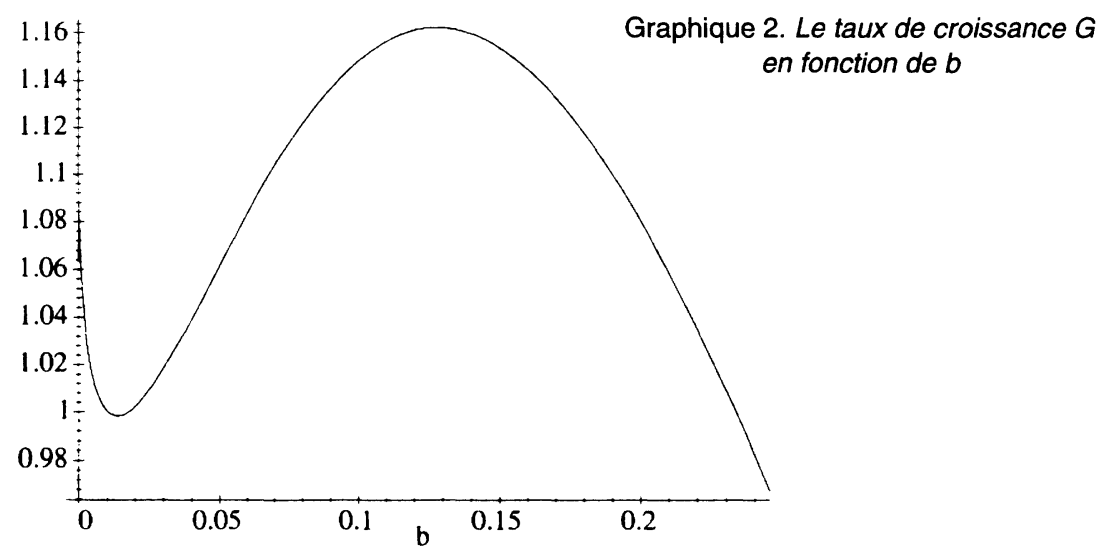

réussit avec certitude que s'il a pu bénéficier des conseils de la banque. On peut supposer pour simplifier qu'il ne réussit pas sans l'aide de ces conseils. Dans ce cas, les projets de grande taille (associés à $\bar{z}$ ) ne seront entrepris que lorsqu'il y aura des grandes banques, alors que les projets de petites taille correspondront à un secteur d'intermédiaition composé de petites banques. Ce dernier cas est associé à un secteur bancaire plus compétitif, donc à une marge d'intermédiation moindre. Cela ne signifie pas qu'un taux de croissance plus élevé accompagne cette concurrence plus intense. Pour $(\underline{b}, z, \bar{b})$, on peut trouver un $\bar{z}$ suffisamment élevé tel que $\overline{\mathrm{G}}>\underline{\mathrm{G}}$, avec

$\overline{\mathrm{G}}=s \cdot(1-\theta) \cdot[1-\mathrm{N}(\bar{b}) \cdot \bar{b}]^{-\theta \cdot(1+\sigma)} \cdot[\theta-N(\bar{b}) \cdot \bar{b}]^{\sigma} \cdot \bar{z}^{1+\sigma}$, et $\underline{G}=s \cdot(1-\theta) \cdot[1-\mathrm{N}(\underline{b}) \cdot \underline{b}]^{-\theta} \cdot(1+\sigma) \cdot[\theta-\mathrm{N}(\underline{b}) \cdot \underline{b}]^{\sigma} \cdot z^{1+\sigma}$, c'est-à-dire :

$$
\bar{z}>\left[\frac{1-\mathrm{N}(\underline{b}) \cdot \underline{b}}{1-\mathrm{N}(\bar{b}) \cdot \bar{b}}\right]^{-\theta \cdot(1+\sigma)} \cdot\left[\frac{\theta-\mathrm{N}(\underline{b}) \cdot \underline{b}}{\theta-\mathrm{N}(\bar{b}) \cdot \bar{b}}\right]^{\sigma} \cdot z^{\sigma}
$$

avec :

$$
\begin{aligned}
& \mathrm{N}(\underline{b}) \cdot \underline{b}=\frac{-\underline{b} \cdot[1+\sigma \cdot \theta \cdot(1-\theta)]+\sqrt{\begin{array}{c}
\underline{b}^{2} \cdot[1+\sigma \cdot \theta \cdot(1-\theta)]^{2}+ \\
4 \cdot \sigma \cdot \underline{b} \cdot \theta \cdot(1-\theta) \cdot[1+\sigma \cdot(1-\theta)]
\end{array}}}{2 \cdot(1-\theta) \cdot \sigma} \\
& \mathrm{N}(\bar{b}) \cdot \bar{b}=\frac{-\bar{b} \cdot[1+\sigma \cdot \theta \cdot(1-\theta)]+\sqrt{\begin{array}{l}
\bar{b}^{2} \cdot[1+\sigma \cdot \theta \cdot(1-\theta)]^{2}+ \\
4 \cdot \sigma \cdot \bar{b} \cdot \theta \cdot(1-\theta) \cdot[1+\sigma \cdot(1-\theta)]
\end{array}}}{2 \cdot(1-\theta) \cdot \sigma}
\end{aligned}
$$

\section{CONCLUSION}

Cet article a présenté un modèle de croissance endogène où les intermédiaires financiers sont en concurrence monopolistique à la Cournot sur le marché des dépôts et sur le marché du crédit. Un secteur bancaire très concentré implique une marge d'intermédiation importante. Cette perte de ressources est absor- 
bée par un coût fixe de fonctionnement des banques, exprimé en termes de masse salariale. Elle correspond un handicap en termes de croissance que l'Allemagne semble avoir surmonté sans problème au cours de la période 1870-1914, et encore maintenant, alors que son système financier reste dominé par quelques grandes banques universelles. Mais ces banques universelles ont comme autre caractéristique d'exercer un pouvoir de contrôle important sur les grandes entreprises, qui vraisemblablement contribue à une hausse moyenne de leur productivité. Cet accroissement de la productivité permet un investissement et des salaires plus élevés, et par conséquence, une hausse de l'épargne. Ces effets peuvent plus que compenser les effets négatifs de la concurrence imparfaite pour une certaine configuration des élasticités des demandes de crédit et de dépôts (déterminant la marge d'intermédiation) et des paramètres de la technologie de contrôle des entreprises reliant l'emploi utilisé dans le secteur bancaire à la productivité des entreprises privées. Lorsque cette technologie de contrôle est peu efficace, les effets négatifs de la concurrence imparfaite prédominent.

En ce qui concerne la relation entre la concentration dans le secteur bancaire et le bien-être ou la croissance, on peut comparer le présent modèle avec celui en équilibre partiel de Yafeh et Yosha [1995]. Dans les deux articles, une dépense d'expertise est nécessaire à l'activité de banque relationnelle. Ce coût est aussi à l'origine de l'imperfection de la concurrence. Dans Yafeh et Yosha [1995], il engendre une hausse du surplus de l'entreprise partagée avec la banque en fonction d'un pouvoir de négociation exogène. Dans cet article, il engendre une hausse de la productivité totale des facteurs et du rendement des prêts. Une condition de positivité des profits incluant un coût d'entrée détermine l'intensité de la concurrence dans les deux modèles. Chez Yafeh et Yosha, la barrière à l'entrée est stratégique : la banque relationnelle déja en place peut interdire l'entrée sur le marché du crédit «non relationnel » en réduisant sa taille par l'extension de son activité de banque relationnelle à un plus grand nombre d'entreprises. Dans le présent article, deux possibilités existent : aucune relation de crédit n'est relationnelle et il y a concurrence parfaite entre les intermédiaires financiers (cas limite de coût fixe nul), ou bien toutes les relations de crédit sont relationnelles. Dans ce cas, la barrière à l'entrée relève de la technologie d'expertise bancaire et un nombre endogène de banques peuvent entrer en concurrence monopolistique à la Cournot. Lorsque l'expertise des banques relationnelles est très efficace, le bien-être (Yafeh et Yosha [1995]) ou la croissance (le présent article) peuvent s'accroître avec une plus faible concurrence bancaire. La distorsion de concurrence entre les banques compense une inefficacité sur le marché des biens et peut même se révéler plus efficace que la concurrence parfaite entre les banques. 


\section{RÉFÉRENCES BIBLIOGRAPHIQUES}

Amable B., Chatelain J.-B. [1995a], « Efficacité des systèmes financiers et développement économique », Economie internationale. 61, p. 99-130.

BERTHÉLÉMY J.-C., VAROUDAKIS A. [1994], «Intermédiation financière et croissance endogène ", Revue économique, 3, p. 737-750.

CAPIE F.H., WEBBER A. [1985], A Monetary History of the United Kingdom, Londres. Allen \& Unwin.

DA RIN M. [1993], « German Kreditbanken 1850-1914 : An Informational Approach », Mimeo, Stanford University.

EDWARDS J., FISCHER K. [1994], Banks, finance and investment in Germany. Cambridge, Cambridge University Press.

FRY M. [1988], Money, Interest and Banking in Economic Development, Johns Hopkins University Press.

GERSCHENKRON A. [1962], Economic Backwardness in Historical Perspective. Cambridge, The Belknap Press of Harvard University Press.

GoLDSMITH R. [1969], Financial Structure and Development, Yale University Press.

KING R.G., LEVINE R [1993], « Finance and Growth : Schumpeter Might Be Right », Quarterly Journal of Economics, 108, p. 717-737.

LEVINE R., RENELT D. [1992], «A Sensitivity Analysis of Cross-Country Growth Regressions », American Economic Review, 83, p. 942-963.

MAYER C. [1988], « New Issues in Corporate Finance », European Economic Review, 32, p. 1167-1188.

NEUBURGER H. [1977], German Banks and German Economic Growth from Unification to World War I, New York, Arno Press.

NeUSSER K., KUGLER M. [1994], «Manufacturing Growth and Financial Sector Development in OECD Countries », Working Paper 1/94, Europa-Universität Viadrina, Fakultät für Wirtschaftwissenschaften.

ROMER P. [1986], «Increasing Returns and Long-run Growth », Journal of Political Economy, 94, p. 1002-1037.

SAINT-PAUl G. [1992], "Technological Choice, Financial Markets and Economic Development », European Economic Review, 36, p. 763-781.

TILLY R. [1986], "German Banking, 1850-1914 : Development Assistance for the Strong ", Journal of European Economic History, 34, p. 710-731.

TILLY R. [1992], "An Overview of the Role of Large German Banks to 1914 », dans CASSIS Y. (ed.), Finance and Financiers in European History, 1880-1960, Cambridge, Cambridge University Press.

YAFEH Y., YOSHA O. [1995], « On the Strategic use of Relationship Banking », communication présentée au XLIV congrès annuel de l'AFSE, Paris.

ZILIBOTTI F. [1994], «Endogenous Growth and Intermediation in an Archipelago Economy », Economic Journal, 104, p. 462-473. 